\title{
Image Processing of Thermal Images using Wavelets
}

\author{
Rolle, Daniel \\ Technische Universität Dresden \\ Institut für Festkörpelektronik \\ 01062 Dresden \\ Germany
}

This poster covers the results of image processing in infrared thermal imaging using wavelets instead of Fourier transformation. Thermal images often contain a plenty of noise to be extracted. The widely used discrete cosinus transformation (DCT) is not able to solve this problem because the noise signal itself doesn't depend on frequencies. In need of using small memories for large amount of data there schould also be taken benefit by using another technique of compression wihtout the well-known artefacts of the JPEG standard at high compression rates.

Wavelet functions are functions that vanish outside a defined intervall. So only this range is of interest. Like any other transformation, the continious wavelet transformation ist defined be the direct product of the signal $x(t)$ and the wavelet-function $\Psi(\mathrm{a}, \mathrm{b}, \mathrm{t})$ :

$$
W_{x}^{\Psi}=\left\langle x(t), \Psi_{a, b}(t)>_{t}=\frac{1}{\sqrt{|a|}} \int_{-\infty}^{\infty} x(t) \Psi\left(\frac{\mathrm{t}-\mathrm{b}}{\mathrm{a}}\right) \mathrm{dt}\right.
$$

The discrete wavelet transformation (DWT) is defined by the convolution of the sampled signal and the wavelet-filter which can be generated by sampling a wavelet-function or a special construction.

A key application of wavelets for infraded images is image compression. Due to the vanishing of wavelets outside the defined intervall the wavelet-basis needed for reconstructing the signal is minor than a cosinus basis at the same loss of quality. So the wavelet-coefficients are rather normally distributed whereas the cosinuns-coefficients are more uniformly distributed in most cases. This causes a highly improved image compression rate due to the entropy of the coefficients.

Denoising can also be achieved by using wavelets. Assuming that the noise is uniformly disctributed on the coefficients as it is in most cases setting small coefficients to zero leads to noise reduction, beacause most coefficients are small, whereas there are only a few crucial coefficients describing the analysed signal.

The discrete wavelet transformation can also be used in order to amplify the contrast in images. Using a reverse threshold technique, where only high coefficients are amplified. This is an selective procedure for pointing out details in images. In comparison to the DCT it only alters coefficients at high frequencies in ranges they themselves are high. So there is no additional side effect like in amplifying high frequencies using the DCT, for the in frequency located wavelets are also located in time, what the DCT doesn't offer. An example can bee seen in the figure below. The original image on the left (a)) has been treated with the DWT for constrast amplifying. The result is shown on the right (b)).

a)

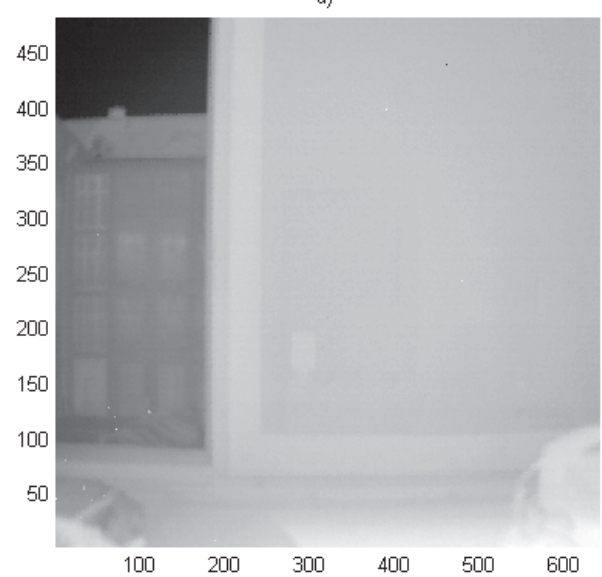

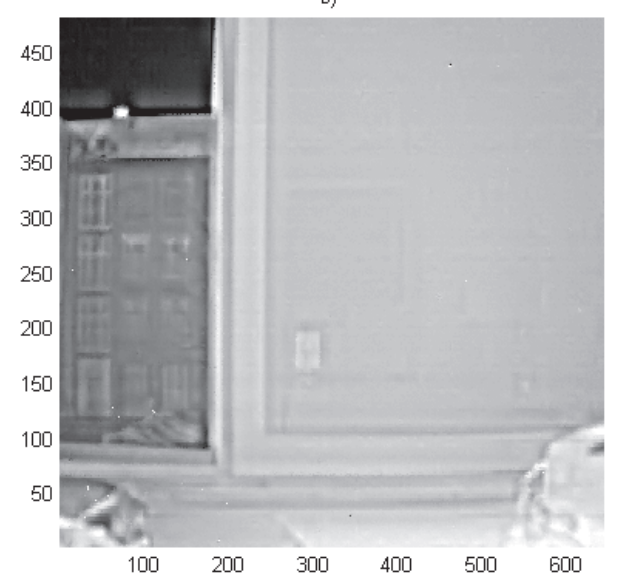

So using a wavelet basis matches all the goals to be achieved such as compression, denoising or contrast amplifying. In comparison to the DCT the DWT offers a plenty of advantages such as higher compression rates, denoising and no side effects in contrast amplifiying. 